\title{
Implementation of the Walrasian correspondence: the boundary problem
}

Citation for published version (APA):

Bochet, O. L. A. (2005). Implementation of the Walrasian correspondence: the boundary problem. METEOR, Maastricht University School of Business and Economics. METEOR Research Memorandum No. 039 https://doi.org/10.26481/umamet.2005039

Document status and date:

Published: 01/01/2005

DOI:

10.26481/umamet.2005039

Document Version:

Publisher's PDF, also known as Version of record

\section{Please check the document version of this publication:}

- A submitted manuscript is the version of the article upon submission and before peer-review. There can be important differences between the submitted version and the official published version of record.

People interested in the research are advised to contact the author for the final version of the publication, or visit the DOI to the publisher's website.

- The final author version and the galley proof are versions of the publication after peer review.

- The final published version features the final layout of the paper including the volume, issue and page numbers.

Link to publication

\footnotetext{
General rights rights.

- You may freely distribute the URL identifying the publication in the public portal. please follow below link for the End User Agreement:

www.umlib.nl/taverne-license

Take down policy

If you believe that this document breaches copyright please contact us at:

repository@maastrichtuniversity.nl

providing details and we will investigate your claim.
}

Copyright and moral rights for the publications made accessible in the public portal are retained by the authors and/or other copyright owners and it is a condition of accessing publications that users recognise and abide by the legal requirements associated with these

- Users may download and print one copy of any publication from the public portal for the purpose of private study or research.

- You may not further distribute the material or use it for any profit-making activity or commercial gain

If the publication is distributed under the terms of Article $25 \mathrm{fa}$ of the Dutch Copyright Act, indicated by the "Taverne" license above, 


\title{
Implementation of the Walrasian Correspondence: The Boundary Problem* Olivier Bochet ${ }^{\dagger}$
}

\author{
June 2005
}

\begin{abstract}
Consider exchange economies in which preferences are continuous, convex and strongly monotonic. It is well known that the Walrasian correspondence is not Nash implementable. Maskin monotonicity (Maskin, 1999) is violated for allocations at the boundary of the feasible set. We derive an impossibility result showing that it is in fact not implementable in any solution concept.

Next, we construct a sequential mechanism based on price-allocation announcements that fits the very description of Walrasian Equilibrium. Imposing an additional domain restriction, we show that it fully implements the Walrasian correspondence in subgame perfect and strong subgame perfect equilibrium. We thus take care of the boundary problem that was prominent in the Nash implementation literature.
\end{abstract}

Keywords: Walrasian equilibrium, preference reversal, double implementation, subgame perfect equilibrium, strong subgame perfect equilibrium.

*This paper is partially based on chapter 2 of my Phd thesis completed at Brown University. I thank Francois Maniquet for comments and discussions on section 3 of the paper. I also thank Roberto Serrano, Rajiv Vohra and William Thomson for helpful discussions and comments on this topic. contact: o.bochet@algec.unimaas.nl

$\dagger$ University of Maastricht and CORE. 


\section{Introduction}

The question of the decentralization of the Walrasian correspondence has received much attention over the past two decades. Hurwicz (1972) first showed that it is not strategy-proof, thereby underlining that dominant strategies is not the right solution concept to use for its implementation. On the other hand, it is well-known (see e.g. Hurwicz-Maskin-Postlewaite (1995)) that it is not Nash implementable either. Maskin monotonicity (Maskin, 1977 and 1999) is violated for allocations that are at the boundary of the feasible set.

In this paper, we raise again the question of the implementability of the Walrasian correspondence. We know that the class of implementable social choice correspondences (henceforth SCCs) rapidly expands when one considers refinements of Nash equilibrium as solution concepts. In their seminal papers, Moore-Repullo (1988) (MR in the sequel) and Abreu-Sen (1990) (henceforth AS) show that Maskin monotonicity is no longer necessary for implementation in subgame perfect equilibrium. In MR, it is shown that the Walrasian Correspondence is implementable in subgame perfect equilibrium when preferences are continuous, convex and monotonic. In contradiction with their claim, we first show that, without further restrictions, the Walrasian correspondence defined over this class of economies is in fact not implementable in any solution concept. Indeed, the Walrasian correspondence is a function of preferences not only inside but also outside of the feasible set. Unless one imposes further domain restrictions, implementation of the Walrasian correspondence is not possible.

After observing that thanks to differentiability, the Walrasian correspondence is implementable in subgame perfect equilibrium, we then proceed to solve the boundary problem in a satisfactory way. ${ }^{12}$

Hurwicz (1979) and Schmeidler (1980) have constructed mechanisms that Nash implement the Walrasian correspondence but in which off equilibrium allocations may award negative quantities to some agents. PostlewaiteWettstein (1989), Giraud-Rochon (2001), Dutta-Sen-Vohra (1995), Tian (1992, 2000) or Sotskov (2003) among others, construct mechanisms that imple-

\footnotetext{
${ }^{1}$ When differentiability is added, the Walrasian correspondence satisfies unchanged contour independence, a sufficient condition for subgame perfect implementation (see Maniquet, 2002).

${ }^{2}$ Obviously, even with differentiability, the Walrasian correspondence is not Nash implementable as long as corner allocations are not excluded.
} 
ments the Constrained Walrasian correspondence. ${ }^{3}$ Papers on non-cooperative bargaining such as Gale (1986, a and b), or more recently Kunimoto-Serrano (2004) provide full implementation of the Walrasian correspondence for economies with a continuum of agents. However, Walrasian allocations at the boundary of the feasible sets are ruled out. Yildiz (2002) covers the two players case, and use assumptions such as uniqueness and interiority of Walrasian allocations.

Therefore, none of the papers cited above take care of the boundary problem. On the other hand, the canonical mechanism constructed in MR and AS would indeed implement the Walrasian correspondence. However, as any general mechanism, it fundamentally lacks economic interpretation. Besides, their game form is complicated and has infinite message spaces. It involves each agent reporting the entire preferences profile. ${ }^{4}$ Parallel to the literature on Nash implementation, we believe that the design of tailormade sequential mechanisms is more appealing. Our aim is to provide an alternative mechanism that is based on the Walrasian notion of allocation and prices and that fits the very description of Walrasian equilibrium.

The important point is that our construction takes care, for the first time, of the boundary problem that was prominent in the Nash implementation literature. Moreover, our mechanism doubly implements the Walrasian correspondence in subgame perfect and strong subgame perfect equilibrium.

The plan of the paper is as follows. Section 2 presents the set-up of the paper. Section 3 deals with our impossibility result. In section 4 , we present the mechanism and the implementation result. Finally, we provide some final comments in section 5 .

\section{The set-up}

\subsection{Economic environments}

There are $L$ infinitely divisible goods and a set of agents $N=\{1, \ldots, n\}$, with $n \geq 2$. The consumption set of each agent $i \in N$ is $\mathbb{R}_{+}^{L}$. For each agent $i \in N, R_{i}$ is the complete and transitive binary relation on $\mathbb{R}_{+}^{L}$ indicating

\footnotetext{
${ }^{3}$ For domains in which Walrasian allocations are interior, the constrained Walrasian and the Walrasian correspondences coincide.

${ }^{4}$ That is, each agent reports his own preferences, as well as the preferences of all the other agents in the economy.
} 
(weak) preferences. The associated strict preference and indifference relations are denoted by $P_{i}$ and $I_{i}$, respectively. The set of possible preferences of each agent $i \in N$ is defined by $\mathcal{R}_{i}$. Denote by $\mathcal{R}=\prod_{i} \mathcal{R}_{i}$ the set of possible preference profiles. A typical preference profile is $R=\left(R_{i}\right)_{i \in N} \in \mathcal{R}$. Each agent $i \in N$ is also characterized by his individual endowment $\omega_{i}>0$. The aggregate endowment is $\bar{\omega} \gg 0 .^{5}$

The only characteristics that vary and that are unknown to the planner are the preferences of agents. An economy is thus a list of preference relation, one for each agent. Formally, an economy is $R=\left(R_{i}\right)_{i \in N} \in \mathcal{R}$. We consider the following two classes of exchange economies.

$\mathcal{R}^{N D}$ : for each agent $i \in N$, every $R_{i} \in \mathcal{R}_{i}^{N D}$ is continuous, convex and strongly monotonic. ${ }^{6}$

$\mathcal{R}^{D}$ : for each agent $i \in N$, every $R_{i} \in \mathcal{R}_{i}^{D}$ is continuous, convex, strongly monotonic and representable by a differentiable utility function.

A (feasible) allocation is a list of bundle $\left(x_{i}\right)_{i \in N} \in \mathbb{R}_{+}^{L n}$ such that $\sum x_{i} \leq$ $\bar{\omega}$. Given an agent $i \in N$, we let $x_{i, l} \in \mathbb{R}_{+}$stand for the quantity of good $l$ received by agent $i$ at bundle $x_{i}$.

The set of feasible allocations $A$ is,

$$
A=\left\{x \in \mathbb{R}_{+}^{L n}: \sum x_{i} \leq \bar{\omega}\right\} .
$$

Let $A_{i}$ be the projection of $A$ onto agent $i$ 's consumption space.

Define by $F$ the set of balanced allocations,

$$
F=\left\{x \in \mathbb{R}_{+}^{L n}: \sum x_{i}=\bar{\omega}\right\} .
$$

Define by $P=\mathbb{R}_{++}^{L}$ the set of strictly positive price vectors.

For each agent $i \in N$, denote by $B_{i}\left(p, z_{i}\right)$ and $\left.B_{i}\left(p, z_{i}\right)\right|_{x_{i} \leq \bar{\omega}}$, the budget set and the constrained budget set, respectively, of agent $i$ at a given price $p$ and bundle $z_{i} \in \mathbb{R}_{+}^{L}$. More formally,

$$
\begin{aligned}
B_{i}\left(p, z_{i}\right) & =\left\{x_{i} \in \mathbb{R}_{+}^{L} \mid p \cdot x_{i} \leq p \cdot z_{i}\right\} \\
\left.B_{i}\left(p, z_{i}\right)\right|_{x_{i} \leq \bar{\omega}} & =\left\{x_{i} \in A_{i} \mid p \cdot x_{i} \leq p \cdot z_{i}\right\} .
\end{aligned}
$$

\footnotetext{
${ }^{5}$ We order vectors with the usual conventions, $\gg,>, \geq$.

${ }^{6} \mathrm{~A}$ preference relation $R_{i}$ defined over $\mathbb{R}_{+}^{L}$ is convex if, for every $x_{i}$ and $y_{i} \in \mathbb{R}_{+}^{L}$ such that $x_{i} P_{i} y_{i}$, we have that $\lambda x_{i}+(1-\lambda) y_{i} P_{i} y_{i}$ for every $\lambda \in(0,1]$.

A preference $R_{i}$ defined over $\mathbb{R}_{+}^{L}$ is strongly monotonic if, for each $x_{i}$ and $y_{i} \in \mathbb{R}_{+}^{L}$, $x_{i}>y_{i}$ implies that $x_{i} P_{i} y_{i}$.
} 
Given an agent $i \in N$, a preference $R_{i} \in \mathcal{R}_{i}$ and a bundle $x_{i} \in X_{i}$, define by $U C_{i}\left(x_{i}, R_{i}\right)=\left\{y_{i} \in \mathbb{R}_{+}^{L}: y_{i} R_{i} x_{i}\right\}$ the upper contour set at $x_{i}$; $L C_{i}\left(x_{i}, R_{i}\right)=\left\{y_{i} \in \mathbb{R}_{+}^{L}: x_{i} R_{i} y_{i}\right\}$, the lower contour set at $x_{i} ; S U C_{i}\left(x_{i}, R_{i}\right)=$ $\left\{y_{i} \in \mathbb{R}_{+}^{L}: y_{i} P_{i} x_{i}\right\}$, the strict upper contour set at $x_{i} ; I C_{i}\left(x_{i}, R_{i}\right)=\left\{y_{i} \in \mathbb{R}_{+}^{L}: x_{i} I_{i} y_{i}\right\}$, the indifference curve through $x_{i}$.

Finally, given a domain $\mathcal{R}$ and $R=\left(R_{i}\right)_{i \in N} \in \mathcal{R}$, an allocation $x^{*} \in A$ is a Walrasian allocation if there exists $p \neq 0$, such that for each $i \in N$, $x_{i}^{*} \in B_{i}\left(p, \omega_{i}\right)$ and $x_{i}^{*} R_{i} y_{i}$, for every $y_{i} \in B_{i}\left(p, \omega_{i}\right) .{ }^{7}$

The Walrasian correspondence $W E: \mathcal{R} \rightarrow A$ associates to each economy $R=\left(R_{i}\right)_{i \in N} \in \mathcal{R}$ its set of Walrasian allocations $W E(R)$.

To conclude, we recall the definition of Maskin monotonicity, the necessary condition $C$ for subgame perfect implementation introduced in MR, as well as a sufficient condition introduced in Maniquet (2002).

Maskin monotonicity: A SCC $f$ satisfies Maskin monotonicity if and only if for each $\left(R, R^{\prime}\right) \in \mathcal{R}^{2}$ and each $a \in f(R)$,

$$
\left[L C_{i}\left(a_{i}, R_{i}\right) \cap A_{i} \subseteq L C_{i}\left(a_{i}, R_{i}^{\prime}\right) \cap A_{i} \text { for each } i \in N\right] \Longrightarrow\left[a \in f\left(R^{\prime}\right)\right]
$$

Condition C: A SCC $f$ satisfies condition $C$ if and only if for each $(R$, $\left.R^{\prime}\right) \in \mathcal{R}^{2}$ and each $a \in f(R) \backslash f\left(R^{\prime}\right)$, there exists a finite sequence of feasible allocations $\left\{a_{0}=a, a_{1}, \ldots, a_{k}, a_{k+1}\right\}$ such that the following is true:

a) For each $l=0, \ldots, k-1$, there exists an agent $j_{l}$ for whom

$$
a_{l, j_{l}} R_{j_{l}} a_{l+1, j_{l}} .
$$

b) There is some particular agent $j_{k}$, with $R_{j_{k}} \neq R_{j_{k}}^{\prime}$, for whom

$$
a_{k, j_{k}} R_{j_{k}} a_{k+1, j_{k}} \text { and } a_{k+1, j_{k}} P_{j_{k}}^{\prime} a_{k, j_{k}} \text {. }
$$

Unchanged contour independence: A SCC $f$ satisfies unchanged contour independence if and only if for each $\left(R, R^{\prime}\right) \in \mathcal{R}^{2}$ and each $a \in f(R)$,

$$
\left[I C_{i}\left(a_{i}, R_{i}\right) \cap A_{i}=I C_{i}\left(a_{i}, R_{i}^{\prime}\right) \cap A_{i} \text { for each } i \in N\right] \Longrightarrow\left[a \in f\left(R^{\prime}\right)\right]
$$

\footnotetext{
${ }^{7}$ Notice that, both in $\mathcal{R}^{N D}$ and $\mathcal{R}^{D}$, preferences are strongly montonic. It implies that at a Walrasian equilibrium $(x, p)$, the price vector $p$ is such that $p \gg 0$.
} 


\subsection{Game-form: definitions and notations}

An extensive game form or mechanism is a game tree with possibly simultaneous moves. More formally, it is defined as an array $\Gamma=(N, T, g)$ where $N$ is the set of players, $T$ a game tree, and $g$ is an outcome function that associates a feasible allocation with each path of play. The set of nodes of the tree $T$ is denoted $S$. The initial node is $s_{0}$. The set of terminal nodes of the tree $T$ is denoted $Z$. Let $M_{i}$ be the set of strategies of player $i$, and let $M_{i}^{s}$ denote the set of strategies available to player $i$ at node $s$. Denote by $M=\prod_{i} M_{i}$, the set of strategy profiles. Suppose the strategy profile $m \in M$ is played. Let $g(m)^{i}$ stand for bundle obtained by agent $i \in N$ at the allocation prescribed by the path induced by $m$, that is, $g(m)$. Let $g(m, s)$ denotes the outcome corresponding to $m$ starting at node $s$. As is common in the implementation literature, we confine our attention to pure strategies.

Given an economy $R=\left(R_{i}\right)_{i \in N} \in \mathcal{R}$, the mechanism $\Gamma$ defines a noncooperative game in extensive game form $(\Gamma, R)$. A subgame perfect equilibrium of $(\Gamma, R)$ is a strategy profile $m^{*} \in M$ such that for all $s \in S \backslash Z$ and for all $i \in N$,

$$
g\left(m^{*}, s\right)^{i} R_{i} g\left(m_{i}, m_{-i}^{*}, s\right)^{i} \text { for each } m_{i} \in M_{i} .
$$

For each $R \in \mathcal{R}$, the set of subgame perfect equilibrium outcomes of $(\Gamma, R)$ is denoted $\operatorname{SPE}(\Gamma, R)$.

A strong Nash equilibrium of $(\Gamma, R)$ is a strategy profile $m^{*} \in M$ such that no coalition of agents have an incentive to deviate simultaneously. That is, for every $m^{\prime} \in M$ and coalition $S \subseteq N$, if $m_{i}=m_{i}^{\prime}$ for each $i \in N \backslash S$, then there is $j \in S$ such that,

$$
g\left(m^{*}\right)^{i} R_{i} g\left(m^{\prime}\right)^{i}
$$

A strong subgame perfect equilibrium of $(\Gamma, R)$ is a strategy profile $m^{*}$ such that for each proper subgame, the profile of strategies is a strong Nash equilibrium in that subgame. For each $R \in \mathcal{R}$ the set of strong subgame perfect equilibrium of $(\Gamma, R)$ is denoted $S S P E(\Gamma, R)$.

An extensive game form $\Gamma$ is said to doubly implement in subgame perfect and strong subgame perfect equilibrium the Walrasian correspondence if and only if

$$
S S P E(\Gamma, R)=S P E(\Gamma, R)=W E(R) \forall R \in \mathcal{R}
$$


We define next the more general notion of implementability.

Implementability: A SCC $f$ satisfies implementability if and only if there exist a solution concept $S$ and a mechanism $G$ such that $G$ implements $f$ via $S$.

We need to introduce one last piece of notation. For each agent $i \in N$, select an $\varepsilon_{i} \in \mathbb{R}_{+}^{l}$ such that $\omega_{i}-\epsilon_{i} \in \mathbb{R}_{+}^{l} \backslash\{0\}$ (note that, for each agent $i$, such an $\epsilon_{i}$ exists since we assumed that $\left.\omega_{i}>0 \forall i \in N\right)$. Define by $\Pi: N \longrightarrow N$ the set of one-to-one functions from the set of agents into itself. Let us define by $f(\pi)$ the composition of the permutations, where $\pi=\left(\pi_{i}\right), \pi_{i} \in \Pi$ for each $i \in N$. Therefore, $f(\pi)=\pi_{1}\left(\pi_{2}\left(\ldots\left(\pi_{i} \ldots\left(\pi_{n}\right)\right) \ldots\right)\right.$ stands for the ordered composition of all permutation of $\pi$. We call $f(\pi)$ a (endogenously determined) protocol. As we shall confine our attention to pure strategies, notice that any agent $i \in N$, by making a unilateral change from $\pi_{i}$ to $\pi_{i}^{\prime}$, can induce any protocol from the composition. The use of permutations is in effect quite similar to an integer game. However, unlike integer devices, the strategy sets are compact. ${ }^{8}$ In our case, it captures an idea of anonymity of the mechanism, in the sense that the equilibria should be independent of protocols. In our framework, ti works as a "king-maker" process since it determines who will lead the game if it goes beyond the first stage. A similar device was used in Serrano-Vohra (1997). Permutations were used first in a different fashion in Thomson (1992, 2004).

\section{$3 \quad$ An impossibility Result}

Given any solution concept, implementability of a SCC requires the following basic property to be satisfied.

Preference reversal: A SCC $f$ has the preference reversal property if and only if for each $R, R^{\prime} \in \mathcal{R}$ and $a \in f(R) \backslash f\left(R^{\prime}\right)$, there exists an agent $i \in N$ and two feasible allocations $x, y \in A$ such that,

$$
x_{i} R_{i} y_{i} \text { and } y_{i} P_{i} x_{i}
$$

Such a property is the most basic requirement for a SCC to be implementable in some solution concept. Consider the situation where $x \in$

\footnotetext{
${ }^{8}$ See Jackson (1992) for a criticism of integer game devices.
} 
$f(R) \backslash f\left(R^{\prime}\right)$ for some $R$ and $R^{\prime}$. If $f$ satisfies implementability, there exists a solution concept $S$ and a mechanism $G$ that implements $f$ via $S$. Precisely, there exist a profile of messages $m$ that is an $S$-equilibrium and that gives $g(m)=x$. Now, if $x \notin f\left(R^{\prime}\right)$ because the only preference reversal that occurs is outside of the feasible set, then $f$ cannot be in fact implemented via $S$ : it is not possible to check information on allocations that are not feasible. Hence, $m$ remains an $S$-equilibrium of $G$ under $R^{\prime}$.

When the preference domain is $\mathcal{R}^{N D}$, the Walrasian correspondence violates preference reversal. The reason stems from the fact that the Walrasian correspondence is a function of preferences both inside and outside of the feasible set. Without further restrictions on the environment, the Walrasian correspondence is not implementable in any solution concept. It is of importance to note that the problem we underline here also pertains to other competitive concept such as the Lindhal correspondence. It is easy to extend Proposition 1 below to the Kolm triangle and Lindhal allocations. The example is available upon request.

Proposition 1: Suppose the domain is $\mathcal{R}^{N D}$ and $n \geq 2$. Then the Walrasian correspondence violates implementability.

Proof: We construct the following example with two agents and two goods. It is easy to see that it extends to cases with three and more agents. There are only two preference profiles $R=\left(R_{1}, R_{2}\right)$ and $R^{\prime}=\left(R_{1}^{\prime}, R_{2}\right)$. The preferences are represented by utility functions as follows.

$$
\begin{aligned}
& u_{1}\left(\cdot, R_{1}\right)=\min \left\{x_{1}+y_{1} ; x_{1}+\frac{1}{3} y_{1}+\frac{8}{3}\right\} \text { and } u_{1}\left(\cdot, R_{1}^{\prime}\right)=x_{1}+y_{1} . \\
& u_{2}(\cdot)=2 x_{2}+y_{2} \\
& \omega_{1}=\omega_{2}=(2,2) .
\end{aligned}
$$

When the profile is $R$, there exists a Walrasian equilibrium $\left(z^{*}, p^{*}\right)$ on the boundary of the feasible set with $z^{*}=((1,4) ;(3,0))$ and $p^{*}=(2,1)$.

The situation is depicted graphically in figure 1 below.

Since $\left(z^{*}, p^{*}\right)$ is not a Walrasian Equilibrium under $R^{\prime}$, implementability requires preference reversal to be satisfied. However, there exists no pair of feasible allocation $x$ and $y$ such that

$$
x_{i} R_{i} y_{i} \text { and } y_{i} P_{i}^{\prime} x_{i} \text { for some } i \text {. }
$$

In conclusion, the Walrasian correspondence is not implementable in any solution concept.

Q.E.D. 


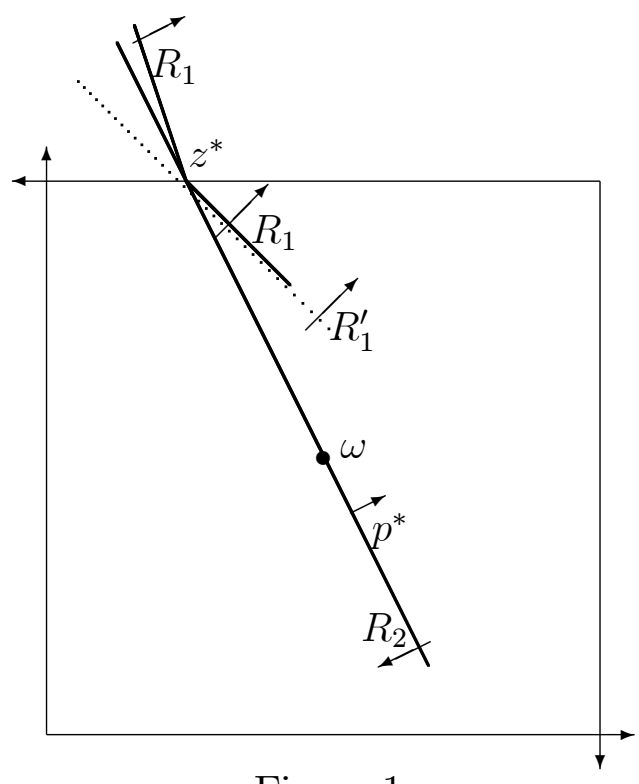

Figure 1

Considering the domain $\mathcal{R}^{D} \subset \mathcal{R}^{N D}$ excludes the case of proposition 1 from the domain. As a consequence, the Walrasian correspondence satisfies unchanged contour independence, a sufficient condition for subgame perfect implementation. ${ }^{9}$ Therefore, if we exclude kinked indifference curves, we do not need further restriction on the environment in order to implement the Walrasian correspondence in subgame perfect equilibrium. In fact, if given two economies an allocation is Walrasian at one preference profile $R$ but not at $R^{\prime}$, differentiability guarantees that local information around that allocation can be used to construct a (feasible) sequence of outcomes as required by condition $C$. This can be seen in figure 2 below.

The allocation $z^{*} \notin W E\left(R^{\prime}\right)$, but we can now identify a sequence of outcome $\left\{z^{*}, x, y\right\}$, as shown in the graph. When differentiability is imposed, the indifference curves going through $z^{*}$ under $R_{1}$ and $R_{1}^{\prime}$ have to be different around $z^{*}$-and inside the feasible set-if $z^{*}$ is no longer Walrasian at $R^{\prime}$.

\section{Taking care of the boundary problem}

We now work with the domain $\mathcal{R}^{D}$ in which preferences are continuous, convex, strongly monotonic and representable by differentiable utility functions.

\footnotetext{
${ }^{9}$ Obviously, even with this domain restriction, the Walrasian correspondence is not Nash implementable since corner Walrasian allocations are not excluded.
} 


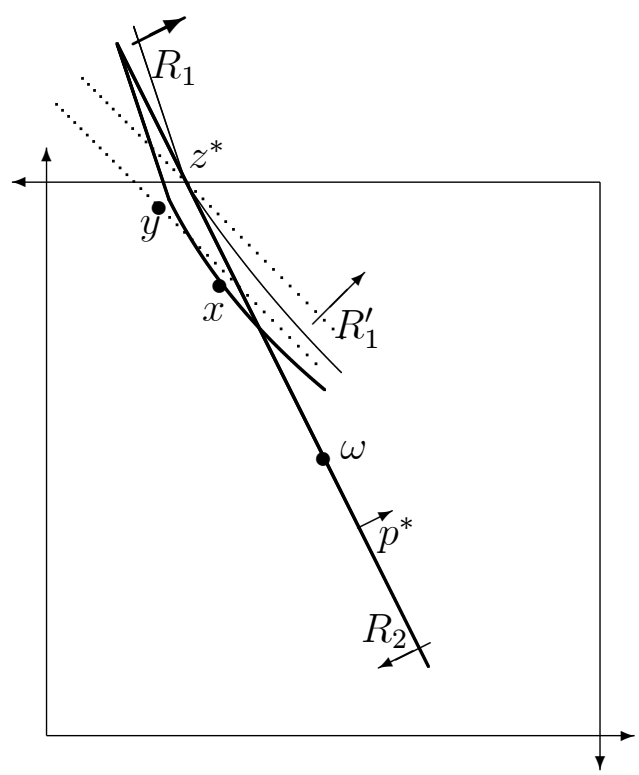

Figure 2

We construct a mechanism that doubly implements the Walrasian correspondence in subgame perfect and strong subgame perfect equilibrium.

The reason for constructing an alternative mechanism to the canonical game form constructed in MR and AS is clear. It is of interest to investigate the design of more tailor-made mechanisms. A simple and more economically appealing mechanism that solves the boundary problem is absent from the implementation literature.

The mechanism we construct has three stages. It involves price-allocation announcements at the first stage, and is thus more reminiscent of a market process. Moreover, it fits closely the description of Walrasian Equilibrium. First, remember that the Walrasian correspondence is implementable in Nash equilibrium over a class of economies in which Walrasian allocations are always interior. It is appropriate to make the game stops at stage 1 if allocations are in the interior of the feasible set. ${ }^{10}$ If, given a price $p \in P$, an interior allocation is not Walrasian, at least one agent would like to obtain a different feasible bundle at that price. Moving along price hyperplanes fits the Walrasian story: no one should prefer any other affordable bundles. This idea was already used, for instance, in Dutta-Sen-Vohra (1995) or Sotskov (2003). Given an interior allocation, the information contained locally

\footnotetext{
${ }^{10}$ Hence, if the domain of economies is such that Walrasian allocations are interior, in equilibrium our mechanism never goes beyond the first stage.
} 


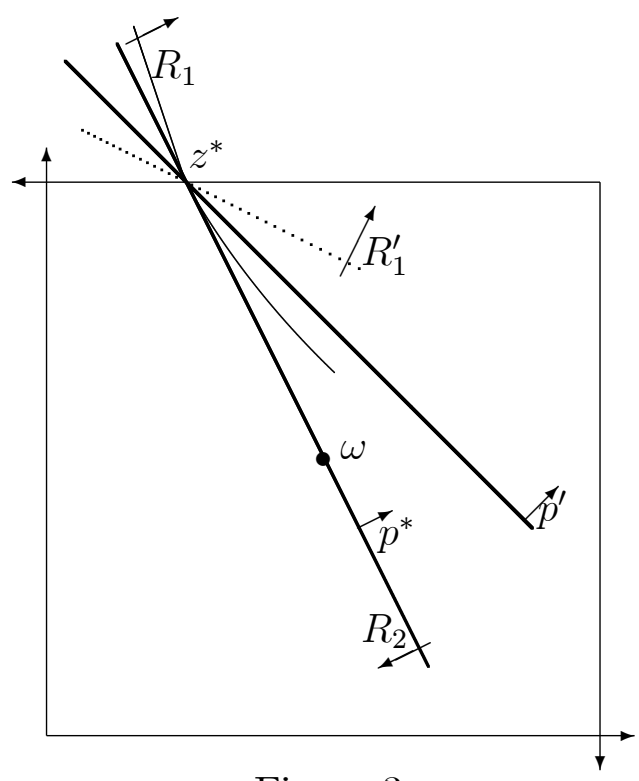

Figure 3

in prices is enough to determine whether or not an allocation is Walrasian. However, when the allocation is on the boundary of the feasible set, this device does not work anymore. Moves along price hyperplanes can lead to infeasible bundles.

Instead, we still rely on the information contained locally in prices, but we use an idea of retrading. At the second stage of the mechanism, an agent can propose a different price vector $p^{\prime}$ to an agent $j$ receiving $x_{j} \gg 0$. This bundle $x_{j}$ plays the role of the new initial endowment for agent $j$. Thus $p^{\prime}$ along with $x_{j}$ generates a new budget set for $j$-hence the idea of retrading. If an allocation $x$ is Walrasian with price $p$, then a different price $p^{\prime}$, with this allocation $x$ as new endowment point will automatically generates agents who would like to retrade. On the other hand, if a boundary allocation is not Walrasian and preferred bundles are infeasible, it is possible to propose a different price vector such that at least one agent-among the agents who receive strictly positive bundles-would not want to retrade. To understand this, take a look at figure 3 above.

The allocation $z^{*} \in W E(R) \backslash W E\left(R^{\prime}\right)$. The price $p^{\prime} \neq p^{*}$ is such that for every feasible bundles $x_{1} \neq z_{1}^{*}$ with $p^{*} \cdot x_{1}=p^{*} \cdot \omega_{1}$, we have that $p^{\prime} \cdot x_{1}<p^{\prime} \cdot z_{1}^{*}$. When agent 1 has preferences $R_{1}$, there exists $y_{1}$ such that $y_{1} P_{1} z_{1}^{*}$ and $p^{\prime} \cdot y_{1}=p^{\prime} \cdot z_{1}^{*}$. However, when agent 1 has preferences $R_{1}^{\prime}$, such a feasible $y_{1}$ does not exist. This indicates that $\left(z^{*}, p^{*}\right)$ is not Walrasian at 
$R^{\prime}$. For if $\left(z^{*}, p^{*}\right)$ was in fact a Walrasian equilibrium at $R^{\prime}$, any $p^{\prime} \neq p^{*}$ such that, for any feasible $x_{1} \neq z_{1}^{*}$ with $p^{*} \cdot x_{1}=p^{*} \cdot \omega_{1}$ and $p^{\prime} \cdot x_{1}<p^{\prime} \cdot z_{1}^{*}$, would create profitable retrading opportunities for agent 1.

The intuition developed with figure 3 is exactly what we use in our mechanism for allocations that are at the boundary of the feasible set.

We shall now present formally the mechanism we use.

\section{Mechanism $\Gamma$ :}

$\forall j \frac{\text { Stage 1: }}{\neq i \text {. If, }} m^{i}=(x, p, \pi)^{i} \in F \times P \times \Pi$ such that $\forall i \in N, p^{i} \cdot x_{j}^{i}=p^{i} \cdot \omega_{j}$

1) $(x, p)^{i}=(\bar{x}, \bar{p}) \forall i \in N$ and $\bar{x}_{i} \gg 0 \forall i \in N$, the game stops and the outcome implemented is $\bar{x}$.

2) $(x, p)^{i}=(\bar{x}, \bar{p}) \forall i \in N$ and $\bar{x}_{j, l}=0$ for some $j$ and $l$, then go to stage 2 .

3) $(x, p)^{j}=(\bar{x}, \bar{p}) \forall j \neq i, i \neq f_{n}(\pi)$ and $m^{i}=\left(x^{\prime}, p^{\prime}\right) \neq(\bar{x}, \bar{p})$.

If $\bar{p} \cdot x_{i}^{\prime}=\bar{p} \cdot \omega_{i}$, then agent $i$ gets $x_{i}^{\prime}$. Agent $j=f_{n}(\pi)$ gets the 0 bundle and the other divide the rest equally. Otherwise if $\bar{p} \cdot x_{i}^{\prime} \neq \bar{p} \cdot \omega_{i}$, each agent $k \in N$ receives his endowment $\omega_{k}$.

4) In all other cases, agent $j=f_{n}(\pi)$ receives $\omega_{j}-\epsilon_{j}$. Each agent $i \neq$ $\left\{f_{n}(\pi), f_{1}(\pi)\right\}$ receives $\omega_{i}$ and agent $k=f_{1}(\pi)$ receives $\omega_{k}+\epsilon_{j}$.

Stage 2: Agent $f_{1}(\pi)$ selects an agent $f_{i} \neq f_{1}(\pi)$ and announces $p^{\prime} \in P$.

1) if $p^{\prime} \neq \bar{p}, \bar{x}_{f_{i}} \gg 0$ and $p^{\prime}$ is such that there exists feasible bundles $y_{f_{i}} \neq \bar{x}_{f_{i}}$, with $\bar{p} \cdot y_{f i}=\bar{p} \cdot \omega_{i}$ and $p^{\prime} \cdot y_{f_{i}}<p^{\prime} \cdot \bar{x}_{f_{i}}$. Go to stage 3 .

2) In all other cases, the game stops and $\bar{x}$ is implemented.

Stage 3: Agent $f_{i}$ chooses between,

$$
q_{f_{i}} \in\left\{q_{f_{i}} \leq \bar{\omega}, q_{f_{i}} \neq \bar{x}_{f_{i}}: p^{\prime} \cdot q_{f_{i}}=p^{\prime} \cdot \bar{x}_{f_{i}}, \bar{p} \cdot q_{f_{i}}>\bar{p} \cdot \bar{x}_{f_{i}}\right\} \text { and } \bar{x}_{f_{i}} .
$$

1) If he chooses $\bar{x}_{f_{i}}$, he gets it. Agent $f_{1}(\pi)$ gets $\bar{x}_{f_{1}(\pi)}+\frac{1}{n-2}\left(\bar{\omega}-\bar{x}_{f_{i}}-\right.$ $\left.\bar{x}_{f_{1}(\pi)}\right)$. If agent $f_{i} \neq f_{n}(\pi)$, then agent $f_{n}(\pi)$ receives 0 and the other agents $j \notin\left\{f_{1}(\pi), f_{i}, f_{n}(\pi)\right\}$ divide the rest equally. Otherwise, if $f_{i}=f_{n}(\pi)$, then agent $f_{n-1}(\pi)$ receives 0 and the other agents $j \notin\left\{f_{1}(\pi), f_{i}, f_{n-1}(\pi)\right\}$ divide the rest equally.

2) If he chooses $q_{f_{i}(\pi)}$, he gets it. Agent $f_{1}(\pi)$ gets 0 and the others divide the rest equally if any.

We can now proceed to the main theorem of the paper. 
Theorem 1: Suppose the domain is $\mathcal{R}^{D}$ and that $n \geq 3$. Then, the mechanism $\Gamma$ doubly implements the Walrasian correspondence in subgame perfect and strong subgame perfect equilibrium.

Proof: We first show that $\operatorname{SPE}(\Gamma, R) \subseteq W E(R) .{ }^{11}$ That is, we proceed to show that if $m$ is a subgame perfect equilibrium of $(\Gamma, R)$, then $g(m) \in W E(R)$. In order to prove the assertion, consider a subgame perfect equilibrium $m$, in which $m_{i}^{1}=(x, p, \pi)^{i}$ and $g(m)=a$. The proof is divided in several lemma.

Lemma 1: $(x, p)^{i}=(\bar{x}, \bar{p}) \forall i \in N$

Suppose not. We have two cases to consider.

Case 1: $(x, p)^{j}=(\bar{x}, \bar{p}) \forall j \neq i$ and $i \neq f_{n}(\pi)$.

First, if $\bar{p} \cdot x_{f_{1}(\pi)}^{\prime}=\bar{p} \cdot \omega_{f_{1}(\pi)}$, agent $f_{n}(\pi)$ gets the 0 bundle. We can construct a profitable deviation for this agent. He deviates by appropriately announcing a permutation so as to be first in the protocol, and a different price-allocation pair. A consequence of such a deviation is that he then receives his endowment majored by a positive epsilon. Since $\omega_{i}>0$ and preferences are strongly monotonic, this is a profitable deviation for agent $f_{n}(\pi)$. A contradiction.

Second, if $\bar{p} \cdot x_{f_{1}(\pi)}^{\prime} \neq \bar{p} \cdot \omega_{f_{1}(\pi)}$, then everyone receives his endowment. But notice that any agent $j \neq i$, by modifying his permutation so as to be first in the protocol and announcing $\left(x^{\prime}, p^{\prime}\right) \neq(\bar{x}, \bar{p})$ could obtain $\omega_{j}+\epsilon_{f_{n}(\pi)}$. Since $\epsilon_{k}>0$ for every $k \in N$ and preferences are strongly monotonic, this is a profitable deviation. A contradiction.

Case 2: One agent $i=f_{n}(\pi)$ disagrees with the other about the priceallocation pair, or more than one agent makes contradictory announcements of a price and an allocation.

In such a case, agent $i=f_{n}(\pi)$ receives $\omega_{i}-\epsilon_{i}$ and each agent $j \notin$ $\left\{f_{1}(\pi), f_{n}(\pi)\right\}$ receives $\omega_{j}$. Any such agent $j \notin\left\{f_{1}(\pi), f_{n}(\pi)\right\}$ could deviate by announcing a different permutation so as to be first in the protocolmodifying his announcement of a price and allocation if necessary-and receive $\omega_{j}+\epsilon_{f_{n}}$. Since $\epsilon_{k}>0$ for each $k \in N$ and preferences are strongly monotonic, this is a profitable deviation for agent $j$, a contradiction.

Therefore, both cases lead to a contradiction with $m$ being a subgame perfect equilibrium.

\footnotetext{
${ }^{11}$ Since $\operatorname{SSPE}(\Gamma, R) \subseteq S P E(\Gamma, R)$, it is enough, for the first part of the proof, to show that $S P E(\Gamma, R) \subseteq W E(R)$.
} 
Lemma 2: If for each $i \in N, \bar{x}_{i} \gg 0$, then $(\bar{x}, \bar{p})$ is a Walrasian Equilibrium

Suppose not. The game stops at stage 1 . The allocation $\bar{x}$, with $\bar{x}_{i} \gg 0$ for each $i \in N$, is the outcome of the game but is not Walrasian given the price $\bar{p}$.

By definition of a Walrasian equilibrium, convexity of preferences and the fact that $\bar{x}$ is an interior allocation, there exists an agent $i$ with preferences say, $R_{i} \in \mathcal{R}_{i}$, and a feasible bundle $x_{i}^{\prime}$ with $p^{\prime} \cdot x_{i}^{\prime}=p^{\prime} \cdot \omega_{i}$, and such that $x_{i}^{\prime} P_{i} \bar{x}_{i}$. Agent $i$ can deviate at stage 1 by appropriately announcing a permutation $\pi_{i}^{\prime} \neq \pi_{i}$ so as to be, say, first in the protocol, as well as $\left(x^{\prime}, \bar{p}\right)$ with $x_{i}^{\prime}$ as identified above. In $x^{\prime}$, agent $i$ assigns, say, $x_{j}^{\prime}=\frac{\bar{\omega}-x_{i}^{\prime}}{n-1}$ to each agent $j \neq i$. Agent $i$ is awarded $x_{i}^{\prime}$, which is strictly preferred. This is a profitable deviation, a contradiction.

As a consequence, if $\bar{x}$ is an interior allocation, it is the outcome of the game and it is Walrasian given $\bar{p}$.

Lemma 3: If $\bar{x}$ is a boundary allocation, then it is the outcome of the game

Suppose not. There exists an agent $i \in N$ for whom $\bar{x}_{i, l}=0$ for some $l$, and the game goes beyond stage 2 . By the rules of the game, one agent $k \in\left\{f_{1}(\pi), f_{n}(\pi), f_{n-1}(\pi)\right\}$ receives the 0 bundle. Consider such an agent $k$. Agent $k$ modifies his permutation, if necessary, so as to be first in the protocol. At stage 2, agent $k$ announces $p^{\prime}=\bar{p}$ and whatever name in the remaining protocol. The game stops with $\bar{x}$ as outcome.

Remember that for each agent $j \in N, \omega_{j}>0, \bar{p} \cdot \bar{x}_{j}=\bar{p} \cdot \omega_{j}$ and $\bar{p} \gg 0$. Thus, it is the case that $\bar{x}_{j}>0$ for each agent $j \in N$. Hence, by deviating, agent $k$ can obtain $\bar{x}_{k}>0$. By strong monotonicity of preferences, this is a profitable deviation. A contradiction.

Lemma 4: If $\bar{x}$ is a boundary allocation, then $(\bar{x}, \bar{p})$ is a Walrasian Equilibrium

Suppose not. $\bar{x}$ is the outcome of the game at stage 2 but $(\bar{x}, \bar{p})$ is not Walrasian. We have two cases to consider.

1) $\bar{x}$ is the outcome of the game but there exists an agent $i \in N$, with preferences $R_{i} \in \mathcal{R}_{i}$, for whom

$$
\left.B_{i}\left(\bar{p}, \omega_{i}\right)\right|_{x_{i} \leq \bar{\omega}} \cap S U C_{i}\left(\bar{x}_{i}, R_{i}\right) \neq \emptyset .
$$

Agent $i$ has a profitable deviation. He deviates at the first stage and modifies his permutation, if necessary, so as to be first in the protocol. At stage 1, he 
announces $\left(x^{\prime}, \bar{p}\right)$ with $\left.x_{i}^{\prime} \in B_{i}\left(\bar{p}, \omega_{i}\right)\right|_{x_{i} \leq \bar{\omega}} \cap S U C_{i}\left(\bar{x}_{i}, R_{i}\right)$ such that $\bar{p} \cdot x_{i}^{\prime}=\bar{p} \cdot \omega_{i}$. For each agent $j \neq i, x_{j}^{\prime}=\frac{\bar{\omega}-x_{i}^{\prime}}{n-2}$. In consequence, the game stops at stage 1 and agent $i$ receives $x_{i}^{\prime}$ which is strictly preferred to $\bar{x}_{i}$ by construction. This case is therefore not possible in equilibrium. The only case left is when there exists an agent $i \in N$, for whom $\left.B_{i}\left(\bar{p}, \omega_{i}\right)\right|_{x_{i} \leq \bar{\omega}} \cap S U C_{i}\left(\bar{x}_{i}, R_{i}\right)=\emptyset$.

2) $\bar{x}$ is the outcome of the game but there exists an agent $i \in N$, with preferences $R_{i} \in \mathcal{R}_{i}$, for whom

$$
B_{i}\left(\bar{p}, \omega_{i}\right) \cap S U C_{i}\left(\bar{x}_{i}, R_{i}\right) \neq \emptyset .
$$

Since the previous case is ruled out, we have that if $x_{i}^{\prime} \in B_{i}\left(\bar{p}, \omega_{i}\right) \cap$ $S U C_{i}\left(\bar{x}_{i}, R_{i}\right)$, then $x_{i, l}^{\prime}>\bar{\omega}_{l}$ for some good $l$. Notice that for this agent $i, \bar{x}_{i} \gg$ 0 . Consider an agent $j \neq i$. Agent $j$ has a profitable deviation. He announces a different permutation at stage 1 , if necessary, so as to be first in the protocol. At stage 2 , he announces $p^{\prime} \neq p$ such that $\left.B_{i}\left(p^{\prime}, \bar{x}_{i}\right)\right|_{x_{i} \leq \bar{\omega}} \cap U C_{i}\left(\bar{x}_{i}, R_{i}\right)=\left\{\bar{x}_{i}\right\}$, -where $\left.B_{i}\left(p^{\prime}, \bar{x}_{i}\right)\right|_{x_{i} \leq \bar{\omega}}=\left\{x_{i} \leq \bar{\omega}: p^{\prime} \cdot x_{i} \leq p^{\prime} \cdot \bar{x}_{i}\right\}$-, and calls agent $i$. The best response of agent $i$ at stage 3 is to choose $\bar{x}_{i}$. Hence, agent $j$ will be awarded $\bar{x}_{j}+\frac{1}{n-2}\left(\bar{\omega}-\bar{x}_{i}-\bar{x}_{j}\right)$. Since $\bar{x}_{k}>0$ for each $k \in N$ and preferences are strongly monotonic, this is a profitable deviation.

Thus, both cases lead to the construction of a profitable deviation, a contradiction with $m$ being a subgame perfect equilibrium. This concludes the first part of the proof.

We have showed that every SPE outcome should be a Walrasian allocation (on the boundary or inside the feasible set). To complete the proof, we prove the opposite direction. That is, we prove that $W E(R) \subseteq S \operatorname{SPE}(\Gamma, R)$. Suppose $\left(x^{*}, p^{*}\right)$ is a Walrasian equilibrium and that the preference profile is $R=\left(R_{i}\right)_{i \in N} \in \mathcal{R}$. Then the following strategies support $x^{*}$ as SSPE outcome of $(\Gamma, R)$.

(i) Every agent $i$ announces $\left(x^{*}, p^{*}, \pi^{I}\right)^{i}$, where $\pi^{I}$ is the identity permutation.

(ii) Let $(\bar{p}, \bar{x})$ be the unanimously agreed price-allocation pair and $f(\pi)$ the composition of permutations at stage 1.

Agent $f_{1}(\pi)$ : 
a) If there exists an agent $f_{i} \neq f_{1}(\pi)$ with preferences, say, $R_{f i} \in \mathcal{R}_{f_{i}}$, and $\bar{x}_{f_{i}} \gg 0$, such that $\left.B_{f_{i}}\left(\bar{p}, \omega_{f_{i}}\right)\right|_{x_{f_{i}} \leq \bar{\omega}} \cap S U C_{f_{i}}\left(\bar{x}_{f_{i}}, R_{f_{i}}\right)=\emptyset$ and there exists $x_{f_{i}}^{\prime} \in B_{f_{i}}\left(\bar{p}, \omega_{f_{i}}\right) \cap S U C_{f_{i}}\left(\bar{x}_{f_{i}}, R_{f_{i}}\right)$ with $x_{f_{i}, l}^{\prime}>\bar{\omega}_{l}$ for some $l$.

Agent $f_{1}(\pi)$ calls agent $f_{i}$ and announces an appropriate $p^{\prime}$ (given the rules of the game) such that $\left.\bar{x}_{f_{i}} \in B_{f_{i}}\left(p^{\prime}, \bar{x}_{f_{i}}\right)\right|_{x_{f_{i}} \leq \bar{\omega}} \cap U C_{f_{i}}\left(\bar{x}_{f_{i}}, R_{f_{i}}\right)$ and $\left.B_{f_{i}}\left(p^{\prime}, \bar{x}_{f_{i}}\right)\right|_{x_{f_{i}} \leq \bar{\omega}} \cap S U C_{f_{i}}\left(\bar{x}_{f_{i}}, R_{f_{i}}\right)=\emptyset$.

b) Otherwise, agent $f_{1}(\pi)$ announces $p^{\prime}=\bar{p}$ and calls agent $f_{2}(\pi)$.

(iii) Agent $f_{i}$ chooses the bundle she prefers between $\bar{x}_{f_{i}}$ and

$q_{f_{i}} \in\left\{q_{f_{i}} \leq \bar{\omega}: p^{\prime} \cdot q_{f_{i}}=p^{\prime} \cdot \bar{x}_{f_{i}}, \bar{p} \cdot q_{f_{i}}>\bar{p} \cdot \omega_{f_{i}}\right\}$. If she is indifferent between $\bar{x}_{f_{i}}$ and any such $q_{f_{i}}$, then she announces $\bar{x}_{f_{i}}$.

The optimality of part (iii) is clear. Agent $f_{i}$ chooses the bundle she prefers. In case of indifference, she agrees with agent $f_{1}(\pi) .{ }^{12}$ Now, notice that agent $f_{1}(\pi)$ is playing a best response at stage 2 . He announces $p^{\prime} \neq \bar{p}$ only if there exists an agent $f_{i} \neq f_{1}(\pi)$ for whom $\bar{x}_{f_{i}} \gg 0,\left.B_{f_{i}}\left(\bar{p}, \omega_{f_{i}}\right)\right|_{x_{f_{i}} \leq \bar{\omega}} \cap$ $S U C_{f_{i}}\left(\bar{x}_{f_{i}}, R_{f_{i}}\right)=\left\{\bar{x}_{f_{i}}\right\}$ and there is a $x_{f_{i}}^{\prime} \in B_{f_{i}}\left(\bar{p}, \omega_{f_{i}}\right) \cap S U C_{f_{i}}\left(\bar{x}_{f_{i}}, R_{f_{i}}\right)$ with $x_{f_{i}, l}^{\prime}>\bar{\omega}_{l}$ for some $l$. By doing so, agent $f_{1}(\pi)$ can obtain $\bar{x}_{f_{1}(\pi)}+\frac{1}{n-2}(\bar{\omega}-$ $\left.\bar{x}_{f_{i}}-\bar{x}_{f_{1}(\pi)}\right)>\bar{x}_{f_{1}(\pi)}$ (by appropriately choosing a price $p^{\prime} \neq \bar{p}$, and calling agent $f_{i}$ ). Whenever this condition is not satisfied, one of the best response of agent $f_{1}(\pi)$ is to announce $p^{\prime}=\bar{p}$ and to call agent $f_{2}(\pi)$. Moreover, given the rules of the game, they cannot be made both better off at stage 3 .

Finally, because every Walrasian allocation is individually rational for each $i \in N, B_{i}\left(\bar{p}, \omega_{i}\right) \cap S U C_{i}\left(\bar{x}_{i}, R_{i}\right)=\emptyset$, the behavior in (i) is also optimal. Any deviation by a coalition will result in the same outcome (if agents just modify their permutation) or in an outcome that is weakly dominated by any Walrasian allocations (obtaining individual endowments in which it is not possible to make coalitions strictly better off). This profile of strategies is a strong subgame perfect equilibrium.

Hence, on the equilibrium path, each agent $i \in N$ announces $(x, p)^{i}=$ $\left(x^{*}, p^{*}\right)$ a Walrasian equilibrium. If for each agent $i, x_{i}^{*} \gg 0$, then the game stops at stage 1 and $x^{*}$ is implemented. Otherwise, it goes to stage 2 where agent $f_{1}(\pi)$ confirms the status-quo coming from stage 1 . The game stops and $x^{*}$ is implemented.

Q.E.D.

\footnotetext{
${ }^{12}$ This situation could happen off the equilibrium path.
} 
Remark 1 In the previous section, we underlined that the issue raised in proposition 1 pertains to the Lindhal correspondence. An example of such a mechanism is available upon request.

\section{Conclusion}

We have shown that, without differentiability, the Walrasian correspondence is not implementable in any solution concept. Imposing differentiability of preferences makes the Walrasian correspondence subgame perfect implementable. We constructed a sequential game form that takes care of the boundary problem. It doubly implements the Walrasian correspondence in subgame perfect and strong subgame perfect equilibrium. It is thus robust to coalitional deviations.

Our mechanism is based on price-allocation announcements and fits the Walrasian story. Moves along price hyperplanes are at the heart of the Walrasian equilibrium concept. Price-taking behavior is not an assumption but a consequence of the rules of the game. In addition, the mechanism could be modified so as to cover the case of the Lindhal correspondence. Our mechanism is thus well-adapted to competitive concepts.

\section{References:}

1. Abreu D., Sen A., 1990. "Subgame Perfect Implementation: a Necessary and Almost Sufficient Condition.". Journal of Economic Theory, 50, 285-299.

2. Dutta B., Sen A. and Vohra R., 1995. "Nash Implementation through Elementary Mechanisms in Economic Environments.", Review of Economic Design, 1, 173-204.

3. Giraud G., Rochon C., 2001. "Consistent Collusion-Proofness and Correlation in Exchange Economies.", Journal of Mathematical Economics, 38, 441-63.

4. Gale D., 1986a. "Bargaining and Competition. Part I: Characterization." Econometrica, 54, 785-806.

5. Gale D., 1986b. "Bargaining and Competition. Part II: Existence." Econometrica, 54, 807-818.

6. Hurwicz L., 1979. "Outcomes Functions Yielding Walrasian and Lindhal Allocations at Nash Equilibrium Points." Review of Economic Studies, $46,217-225$. 
7. Hurwicz L., Maskin E. and Postlewaite A. 1995. "Feasible Nash Implementation." in Ledyard J.O. (ed), The Economics of Informational Decentralization. Complexity, Efficiency and stability. Kluwer Academic Publisher.

8. Kunimoto T., Serrano R. 2004. "Bargaining and Competition Revisited." Journal of Economic Theory, 115, 78-88.

9. Maskin E., 1999. "Nash Equilibrium and Welfare Optimality." Review of Economic Studies, 66, 23-38.

10. Moore J. and Repullo R., 1988. "Subgame Perfect Implementation." Econometrica, 56, 1191-1120.

11. Postlewaite A., Wettstein D., 1989. "Feasible and Continuous Implementation." Review of Economic Studies, 56, 603-611.

12. Schmeidler D., 1980. "Walrasian Analysis via Strategic Outcome Functions." Econometrica, 48, 1585-1593.

13. Serrano R., Vohra R., 1997. "Non-Cooperative Implementation of the Core." Social Choice and Welfare, 14, 513-525.

14. Sotskov A.I, 2003. "Characterization of Competitive Allocations and the Nash Bargaining Problem." in Sertel M. and Koray S. (eds) Advances in Economic Design. Springer Verlag.

15. Thomson W., 1993. "Divide and Permute and the Implementation of Solutions to the Problem of Fair Division." Forthcoming in Games and Economic Behavior.

16. Tian G., 2000. "Feasible and Continuous Double Implementation of Constrained Walrasian Allocations." Annals of Economics and Finance, 1, 19-32.

17. Tian G, 1992. "Implementation of the Walrasian Correspondence without Continuous, Convex, and Ordered Preferences." Social Choice and Welfare, 9, 117-130.

18. Yildiz M., 2001. "Walrasian Bargaining.", Games and Economic Behavior, 45,.465-487. 